\title{
Study of Multiliteracy Pedagogy in College English Teaching
}

\author{
Zheng Gu \\ Humanities Department \\ Shandong Management University \\ Jinan, China
}

\begin{abstract}
The paper intends to analyze and construct the teaching and learning modes of the college English by exploring the new framework of multiliteracy pedagogy and integrating the explicit instruction with the transformation of practical teaching methods. The paper deals with the prior designs, the process of designing and redesigns in a progressive way and focused on the application of college English teaching reform with the intention of improving the students' the multiliteracy capability under multimodality language learning environment.
\end{abstract}

Keywords-multiliteracy pedagogy; multimodality; design learning

\section{INTRODUCTION}

In the reform and exploration of the teaching modes of college English, the cultivation of students' English ability has always been the focus of attention of English teaching. The 21st century is an era of multi-cultural diversity and rapid development of information. Education presents a trend of diversification and information, which means the change of education method and education mode as well as the breakthrough of traditional single education environment. In social communication, language is no longer dominant, and images, colors, music and other visual symbols play an increasingly prominent role. A new multimodal expression infiltrates all aspects of social life (Hu Zhuanglin, 2006), which also provides a new idea for foreign language teaching. Multimodal teaching is a new teaching theory which has been explored by domestic and foreign experts in teaching research and practiced for a long time. This theory complies with the diversified development of the technology of social communication. The traditional college English teaching mainly uses the paper textbooks and materials as the media, while the multimodal English reading and writing course combines the characters, animations, pictures, sounds and other symbols to create a learning situation. The multimodal English reading and writing course teaching mode is in line with the new development of foreign language teaching, which can fully mobilize the initiative of students in language learning and cultivate their cognitive ability. At the same time, it is conducive to the comprehensive improvement of students' diversified reading and writing ability, and it provides a new perspective for the establishment of a new and efficient college English teaching mode.

\section{Multiliteracy CAPABILITY}

The concept of multiliteracy capability for the teaching and academic discussions is proposed by New London Group linguists from the United States, Britain, Australia in 1994. They think the culture exists in a variety of forms through multiple symbol system, such as language and words, visual images, sound, while people want to transfer the hearing, sight, touch, such as a variety of channels to acquire knowledge. On this basis, the new London group proposed the multivariate reading teaching method in 1996. It overcomes the disadvantages of the traditional languagecentered teaching mode, using various symbolic resources such as network, picture and voice to learn language and culture, and cultivates the ability of learners to creatively use information communication technology and critically understand and acquire the information provided by multiple media. The method emphasizes the use of various symbolic resources to acquire language and culture, and puts the importance on the negotiation of language and cultural differences in society for learners to use language in work, public and private occasions

There are two main reasons for the transfer of learning objective from listening, speaking, reading and writing ability to multimodality reading and writing ability. First, globalization and diversification have caused the diversification of meaning, which makes the existing way of meaning expression using language as a medium difficult to be overwhelmed. Second, the development of modern technology and modern network technology has forced people to learn to use new media to express and interpret meanings. On the surface, this transformation mainly requires students to learn how to communicate with modern multimedia technology, including selecting appropriate media and combining media to express and interpret meanings. Students need to develop their language reading and writing skills as well as their media literacy and technical literacy skills. As the demand for multimedia literacy capability has increased, its related capabilities have also been highlighted.

\section{THE FRAMEWORK OF MULTILITERACY PEDAGOGY}

In traditional theory, Saussure (1915:67) regarded semiotics, symbols as a whole composing of signifier and 
being signified. Kress (2003:38) believes that as a language symbol, a word is not a symbol as a whole, but a signifier. The process of design is to design the existing symbols according to the meaning expressed in the context and produce a new text. The meaning of symbols requires the designer to refer to the design meaning in the design and the listener to fill the meaning in the interpretation. Each design process changes its meaning on the original basis, which is a meaningful manufacturing process. With the change of symbolic meaning, the original state of the symbol has changed, and the symbol system of the sender has also changed. From this point of view, the symbols produced by design are new, so in the new multivariate reading and writing process, creation is a normal and frequent phenomenon. Under the guidance of social semiotics theory, the framework of multivariate literacy theory includes the following main theoretical concepts.

First, in the construction of multimodal discourse, each mode has a specific function in the overall meaning construction, which results in "modal specializations". For example, written language is more conducive to represent a series of events, while images are more conducive to show the relationship between components in space. In addition, the amount of information loaded by different modes is called functional load. In textbook teaching, the main functional load is carried by written language, while in PPT courseware teaching, the main functional load is carried by spatial layout, special shape, image and animation.

Second, material that reflects the mode has modal effects in using the body to accept and produce meaning. Each mode is associated with a human perception system, such as sound and hearing, motion and touch, and through different sensory systems there are different ways of expressing meaning. This results in a difference in the availability characteristics of different modes.

Third, the human is a new symbol and the existing symbol resource is enough to show the new meaning, such "transformation" and "China Unicom" has become a key concept. The transformation is carried out within the same mode. Unicom represents that the meaning usually represented by one mode or one set of modes is represented by another or another set of modes, which is similar to the "Synaesthesia" in the study of rhetoric. For example, to change a short story written in written form into a comic strip involves changing the meaning of the written form into an image. Connection is a basic mode of human communication. This is a basic concept of multimodality design.

Fourth, there is no material representation for knowledge and information, which must be fixed for it through the material entity. This is the process of using any mode, using any position, etc.

Finally, the structural elements of each mode take a place in the discourse, called "site of appearance". These locations have their own rules and order, which in turn have an impact on the context in which they appear. For example, in the past, page logic dominated, written language dominated the page, and graphic graphics were subordinate to written language. Now, screen logic reigns, images dominate the screen, and written language is subordinate to images. In this case, the screen logic also affects the page logic, on the page, more and more images.

\section{Designs of Multiliteracy Pedagogy}

Design learning is "learning by design", and through the study of memory, through training, learning through practice to complete the task, self-service platform learning concepts such as compared to its concept is advocated by the new London group (1996) and, later became a main theoretical basis of cultivating the ability of multivariate, speaking, reading and writing.

Design learning based on design theory has made many new developments than common learning concepts. First, traditionally, the learning process has been regarded as a passive acceptance process, that is, what knowledge teachers provide and what students learn. In the new design theory, learning is not passive implementation and acceptance, but active design. Teachers only provide learning conditions and requirements. Students achieve learning goals through design. Learners become active creators of meaning. Second, design learning has changed the previous single-mode learning, integrated various resources, given full play to the advantages of various resources, and improved learning efficiency and effect. In this process, students need to choose the appropriate mode or mode combination to realize the meaning. Thirdly, design learning combines knowledge learning and ability development. Learners must firstly know the significance potential of various modes. Then, they need to be able to make meanings by themselves. Fourthly, students' learning ability in the interdisciplinary is enhanced. Human learning has always been a super-discipline. In order to effectively control knowledge, different disciplines have been divided and students from different disciplines have been allowed to specialize in the knowledge of this discipline, which to a certain extent limits the development of students and the improvement of their overall quality. However, in design learning, students must make full use of the relevant knowledge of each discipline and choose various modes to realize the significance of communication, which is conducive to students' development of the ability to control the knowledge of the super-discipline and improve their comprehensive quality. Students need to have a stronger ability to analyze, to evaluate and criticize. When more modes become alternative means of meaning expression, students need to develop the ability to choose, not only to know what to choose, but also to distinguish the advantages and disadvantages so as to select the most effective way of communication to create meaning and achieve the purpose of communication. Finally, innovation becomes the norm in design learning. Although the design process is largely a process of utilizing existing resources, these resources are always used in the new context to realize new communication tasks, and new combinations are also needed to create new meanings, so that existing designs can be transformed into new designs and thus become new existing designs. 


\section{A. Design Learning Objectives}

The design concept in the theory of multimodal discourse analysis is not only related to but also different from the general design concept.Consciously planning and sketching the meaning of something has to be done in the theory of multimodal discourse analysis.It is a concept that makes full use of the communicative mode to carry out effective communication

We organize, and form a modal combination of the whole, the most effective completion of communication tasks. In terms of the relationship between design and communication factors at different levels, design is the process of using all symbol modes or combination of symbol modes as symbol resources, and it is the way to realize discourse in a certain communication context. To use a metaphor, design can be compared to drawings. The object of design can be compared to a house. This is similar to architecture, which is to organize existing resources into a framework for the production of drawings. In addition, the design of multimodal discourse analysis is different from ordinary design, which is an important aspect of the concept of the general design. It is always confined to the coding phase, which is an active process. The design of multimodal discourse analysis includes coding phase and also include the decoding stage, namely the learning phase.

New media and new technology have led to an explosion of knowledge, making it hard for students to learn in the traditional way and choosing useful information in the vast field of knowledge. In this way, knowledge control ability and information classification ability become more important, which is "information literacy ability". Correspondingly, students must develop and use the new social environment and be able to adjust to their "sociocultural reading and writing ability" timely in the new situation. The third thing that is related to this is that students need to develop the ability to criticize. They have the ability to analyze, identify and evaluate all kinds of knowledge and information, and can relate what they have learned to the learning goals and communicative needs. Last but not least, the development of "innovation capacity" plays an important part. With the rapid development of science and technology, students should learn to creatively choose and utilize existing resources or create new resources as well as to create new meanings and transform existing resources into new ones. Therefore, they must develop their innovation ability or transform their reading and writing ability.

\section{B. Design Learning Contents}

What can you learn to develop multiple literacy skills? The new London team studied this problem in 1996 and proposed five main modes or modality combination types: language mode, auditory mode, visual mode, spatial mode and body potential mode. Each mode consists of different modal components. Language modal elements include expression mode, vocabulary, metaphor and substance, process standardization, information structure, microcoherence relationship, macro-coherence relationship, etc. The auditory modalities include music, sound effects, etc.
Visual modal elements include color, perspective, vector, foreground and background. Spatial modal elements include ecosystem, geographical location, architectural significance, etc. The interaction and cooperation among five modality components, including behavior, body shape, gesture, feeling, emotion, body movement, spatial relationship, etc., are integrated and utilized in multimodality.

\section{Procedures of Design Learning}

First of all, teachers should change the teaching mode from teaching knowledge mainly in class to pay attention to the teaching mode of constructing knowledge independently in the network environment. Secondly, teachers should make full use of multimedia network resources to establish a diversified learning model of discourse.

The multivariate epistemology teaching method takes the design construction meaning as the teaching idea and social semiotics as the theoretical basis. Design theory first put forward by new London group, including three parts, the existing design (the available design), the design process (designing) and design (the redesigned). Existing design is an existing meaningful resource. The design process is the work of utilizing existing resources in the symbolic process. It emphasizes that the construction of meaning is a positive and dynamic process, rather than being controlled by static rules.

In the existing college English textbooks, each unit contains one intensive reading and two extracurricular reading texts. For example, the intensive reading text is first read through the text, and then interpreted in the context. Finally, the topic of the text is taken as the center for discussion or debate, so that students can express critical views on what they have learned, so as to cultivate and develop students' ability to analyze, explain, summarize and explain problems.

Thematic teaching is one of the popular teaching modes in college English. Therefore, teachers can arrange students to make PPT works related to the theme and present them in class. Of course, teachers should give "clear guidance" to students in advance, so that students can understand that every symbol, whether text, image or sound, is a meaning resource. In the process of meaning expression, sometimes a symbol mode needs to rely on other symbol modes to complete or better complete information and meaning transmission.

This model aims to try a new writing ability. The students can be assigned to design electronic tabloids. Make writing a multimedia work with both picture and text and sound, provide a wide space for the expansion of students' writing content, and train students' abilities of scientific and technological literacy, media literacy, cultural literacy, political literacy and critical literacy comprehensively. Since teachers have become the developers of teaching discourse in the new education environment, both the teaching process and courseware production try to use original audio and video materials and text materials to create a relatively real language atmosphere. In addition, we provide students with high-quality learning websites, so as to expose them to the 
multicultural world of knowledge and reading, so as to enhance their awareness of multicultural communication and cross-cultural communication.

Multimodality reading teaching variability and students' initiative are the essential part. Cultural and linguistic diversity is not only a social resource but also a teaching resource. When learners learn different languages, different styles and different methods can exist at the same time. They have acquired a lot of metacognitive abilities, and have used these abilities to make a critical response to the communication between classmates. In teaching, the differences among students from different classes, races and countries are positively recognized. Such differences are negotiated among students to make up for each other. Students can take this opportunity to expand their cultural and language skills so that they can access greater social and cultural resources. At the same time, learners have developed subjectivity in the learning process, and constructed their self-identity, personal values and world view.

The teaching method of multivariate epistemology attaches importance to the application of various new communication methods and media brought about by the progress of science and technology in English classroom and does not ignore the role of multimedia in learners' real life, which sees the influence of modern technology on language learning. For example, the Internet is changing the way of teaching and learning, allowing people to conduct knowledge construction activities in virtual non-physical space, which can be said to be a total subversion of traditional teaching and learning methods. Network brings new learning environment, of course, it is necessary to think about how to implement new reading education. Only when the new reading ability is diversified can we use technology to actively cope with the new life style.

The multivariate epistemology method emphasizes critical reflexive language learning. It pays attention to the relationship between language and power, and opposes students' sitting on their hands in class, taking full advantage of what teachers teach, and believing in the authority of textbooks. It holds that most texts have specific ideology and views, and learners must cultivate the habit of selfreflection in the process of learning, instead of blindly accepting and internalizing ideas from textbooks, teachers or peers. At the same time, teachers should provide learners with socialized language learning activities different from traditional ones, help them reflect on themselves and think about the external environment, enable learners to understand various modal discourses from various information transmission methods and information networks develop critical thinking skills and enhance cross-cultural awareness.

\section{The ApPlication of Multiliteracy Pedagogy}

The teaching method of multiple knowledge pay attention to the variability of classroom teaching and the initiative of students. It pays attention to the application of various new communication methods and communication media brought about by the progress of science and technology in English classroom, not ignoring the role of multimedia in learners' real life, and sees the impact of modern technology on language learning. For example, the Internet is changing the way of teaching and learning, allowing people to conduct knowledge construction activities in virtual non-physical space, which can be said to be a total subversion of traditional teaching and learning methods. Network brings a new learning environment, of course, it is necessary to think about how to implement new motivational strategies to stimulate students' learning enthusiasm.

\section{A. Implementing Motivational Strategies}

The interaction between teachers and students is a great source of motivation for students to learn. Due to the restriction of limited class hours and too many students in the class, the interaction between teachers and students in college English class is often insufficient to meet the needs of students. Teachers and students can effectively make up for this deficiency by maintaining communication and interaction through online platforms after class. In this sense, the network interaction between teachers and students extends and sublimates the interaction between teachers and students in class. It shows that teachers and students communicate and communicate in an equal, coordinated and harmonious way. The relationship between teachers and students is a dynamic, two-way form, not a static, one-way form. It is a process of building knowledge and collaborative meaning. Teachers should always remember that computer and network tools are only the medium of communication, and students are the objects of communication. In the process of communication, students' emotions and needs should be taken into account, and interaction should be promoted and maintained by means of encouragement, comments and sharing. The network environment is conducive to the weakening of the identity of teachers and students. Teachers can guide the direction of communication and promote the progress of communication. The main purpose of network interaction is to strengthen the communication between teachers and students and promote students' learning. Teachers should try their best to use the dialogue mechanism to promote students' knowledge construction and meaning negotiation.

\section{B. Interacting by Using Online Tools}

There are many ways of online interaction between teachers and students, such as E-mail, BBS, QQ, online chat room, blog, and Internet message, etc.. Each has advantages, such as QQ can quickly transfer files; BBS is more convenient for teachers and students to post posts or online messages. Teachers can choose according to the specific circumstances. With the aid of the network interactive tools, not only can teachers across time and space limit, maintain contact with the student, to strengthen the guidance of learning, students can also drive the communication between each other and learning. They can even build a small network learning community in class; let the students have more chance to experience the advantages of collective learning, enhancing the sense of belonging. 
Teachers' teaching beliefs and attitudes determine the implementation of task teaching, to what extent "teachercentered" or "student-centered" should to consider. Traditional methods usually specify subjects for teachers, set task goals, select materials and suggest learning process. In this case, the "need for autonomy" to stimulate students' inner motivation is not satisfied. Students can choose what to learn and how to learn in "student-centered" teaching. Of course, this choice needs to be made under the guidance of teachers or through consultation with teachers.

\section{Changing the Role of the Students and the Teachers}

In multimodalility classroom implementing task-based teaching, the most important thing for teachers is to play the role of guide and monitor. This effect runs through the whole teaching process, especially in the stage of task implementation. Teachers need to keep an eye on the progress of the task and provide guidance and help when students encounter obvious and insurmountable difficulties. But at the same time, attention should be paid to avoid excessive intervention, leaving students as much space as possible to think independently, negotiate with each other and seek solutions to problems. After the completion of learning activities, it is necessary for teachers to make targeted evaluation and feedback on the completion of tasks, so as to consolidate students' knowledge, promote students' reflection, and provide references and experience for later learning. Improper positioning or inadequate concentration of the role of teachers will often make students feel helpless, disoriented and even misguided. The role of teachers and the extent to which they can perform are related to their teaching belief and attitudes, which is also influenced by their computer skills.

Students are intrinsically motivated when they feel competent for a task. Students' computer skills and language skills are crucial to task selection and design. Task requirements far beyond students' ability level can only make students fear and resist. The teacher must have a clear understanding of the students' ability level before carrying out the task. If students lack specific skills, they can either adjust their tasks or provide adequate training to help them master these skills (Hubbard 2004). It is worth noting that, on the premise of guaranteed training or guidance, tasks that are higher than students' current abilities will not affect students' confidence, but will stimulate their curiosity and interest.

The objectives set by the task should also meet the learning needs of students, so that students can feel the realistic significance of participating in learning activities. In addition, task-based learning in classroom usually requires students to interact and communicate with each other. The lack of individual communicative intention often affects the establishment of group cooperation relations and the formation of a sense of belonging.

\section{The Integration of Multimodal Classroom}

The integration of computer and network not only provides a lot of convenience and abundant resources for teaching, but also complicates the design of task-based learning activities. The computer ability of teachers and students has been challenged, and the limitations and problems of the multimodal environment have increased the difficulty of design. Therefore, the design of multimodal classroom task-oriented learning activities should start from the analysis of teaching subjects and the evaluation of network environment, namely, to evaluate teachers' computer ability, students' language ability and computer ability, and to confirm the status of software and hardware facilities, the availability of network resources/tools and technical support. Based on the assessment results, teachers and students negotiate the selection or design of tasks, and jointly determine the objectives, themes, materials and estimated completion time of tasks. It must be noted that due to the lack of experience, the participation of students can only be conducted under the guidance of teachers. The participation degree and participation mode are also determined by teachers according to the actual situation. It not only ensures that students have a certain degree of autonomy in their own learning activities, but also does not lose their direction and make inappropriate choices or decisions. During the implementation phase, students perform tasks in groups and report to the class on the completion of tasks and results or conduct group communication and discussion. Teachers' role in this process mainly includes the supervision of students to complete the task according to the requirements of the target, encourage and assist "weak" students more involved in group activities, to give meaning to promote construction language tips (only in the circumstances the exchange was blocked and unable to "save" or when the students take the initiative to help students), can provide technical support or seeking "foreign aid" for students. After the completion of the task, the teacher makes evaluation and feedback on the implementation of the task and the content of students' report or discussion, so as to inspire students to reflect on the learning process and summarize the successes and failures. This link is very important and provides valuable experience and reference for future task design. Finally, students consolidate the language difficulties in task implementation through practice.

The goal of foreign language classroom teaching is to complete the prescribed teaching objectives and tasks within the prescribed time. Teachers have both the responsibility to teach students and the power to manage them. Traditional classroom layout for visual mode, it determines the teaching environment, and determines the relationship between the teacher and the students' power and role: podium, blackboard. The teacher is the student's visual object, establishing the teacher's desk and platform of authority and responsibility for the teacher on the platform overlooking the students. The blackboard plays the role of the teachers' main tool of this authority. The teaching of multiple modes also includes some other modes. Although the classroom layout realized by the visual mode determines the position and role of teachers, it only provides background information and conditions and plays an auxiliary and strengthening role in the auditory mode. From multiple modal point of view, teacher's dominant, accounted for about $60 \%$ to $80 \%$ of the whole discourse, and the teacher's 
discourse is mainly for students to accept the object, it puts forward very high requirements about the quality of the teachers discourse: significance for accurate, requires correct grammar, vocabulary required precision, requires accurate, moderate speed requirements. This is the reason why the evaluation of the teaching quality of college English classes usually requires a high level of teachers' oral language. At the same time, the teacher's sound loud, high and low tone, tune, rhythm, stresses have a great impact on the teaching effect. Therefore, within each mode, various modes coordinate with each other and play a role of optimizing, supplementing and strengthening the oral discourse mode. Teachers themselves supplement and strengthen their own discourse through visual modes, including using gestures to express rhythm, simulating what they say and concepts, and displaying the importance of what they say with changes in facial expressions. Teaching is different from ordinary daily communication, which is usually limited to communicating information with little difficulty, and usually does not require memorization. And the information that the teaching communicates always has difficulty, and not only is the information transmission, more importantly is the student ability of cultivation, teaching the student to obtain the related language ability. Therefore, teachers should use certain tools, such as blackboard and PPT. These tools and media can not only strengthen the teaching content, for example, reproduce key knowledge and words with blackboard, provide the outline of knowledge with PPT, but also supplement it, for example, provide background information and specific details with PPT, provide real scenes with pictures, and provide real process with animation. Discourse communication is bilateral; especially the cultivation of students' ability requires students to speak in person to improve their ability of speaking foreign languages.

\section{CONCLUSION}

In conclusion, the successful design of college English teaching model lies in how to construct a learning community to adapt to the college English multimedia environment, how to evaluate the role between the teachers, students and the multimedia, how to make reasonable distributions between the role. These implementation would help the teachers and students with the aid of multimedia mutual participation in multivariate improve process of literacy, making teachers and students form the good interaction between students and students, the classroom mode as well as the process, developing direction, diversified development, timely provide students with the teaching of remedial measures, and ultimately promote the development of students' English learning ability. Its main innovation lies in the following aspects: it designs a design based on teaching mode of multiple intelligence, explores the influence of multiple intelligence on students' learning and motivation, and tests the feasibility of multiple intelligence in the field of college English teaching. In a comprehensive way, this teaching method involves the whole process of teaching and all aspects, not only knowledge learning, but also the improvement of skills and overall quality. It involves not only the teaching process, but also the learning tasks and learning ideas of students after teaching. It involves not only what you learn, but also how you learn. From the perspective of introspection, this teaching method is not only to students to actively participate in social practice and social activities, by attending these activities, automatic gain communication skills, and they have to rethink the practice activities. Through these they learn how to contact and compare with the original resources, reconstruct their knowledge structure and ability framework.

\section{REFERENCES}

[1] Halliday, M. A. K. \& Matthiessen, C. An Introduction to Functional Grammar (3 ${ }^{\text {rd }}$ ed $)[\mathrm{M}]$. London: Arnold, 1994.

[2] Jonassen, D.H. Evaluating constructivistic learning. Education \& Urban Society, 1991, 24(4):466 477.

[3] Kalantzis, M. \& Cope, B. Multiliteracies: Literacy Learning and the Design of Social Future [M]. London: Routledge, 2000: 26.

[4] Kalantzis, M. \& Cope, B. Language Education and Multiliteracies [A]. In May, S. \& Hornberger, N.H.(eds.).Encyclopedia of Language and Education [C]. Boston: Springer Science + Business Media, LLC 2008: 195-211.

[5] Kendrick, M. \& McKay, R. Researching Literacy with Young Children's Drawnings [A]. In Narey M. (ed.). Making Meaning [C]. Boston: Springer, 2009: 53-70.

[6] Kress G \& Van Leeuwen T. Reading Images: The Grammar of Visual Design [C]. London: Routledge, 1996.

[7] Kress G. Multimodality [C] //Cope B, KalantzisM. Multiliteracies. London: Routledge, 2000.

[8] Kress, G. et al. Multimodal Teaching and Learning: The Rhetorics of The Science Classroom [M]. New York: Continuum, 2001.

[9] Kress G. Literacy in the New Media Age [M]. London: Rouledge, 2010: 1 .

[10] Leeuwen. Introducing Social Semiotics [M ]. London: Routledge, 1996 